\title{
Image Fusion in SIMS-based Correlative Microscopy: Methodology and Applications
}

\author{
Jean-Nicolas Audinot ${ }^{1}$, Florian Vollnhals ${ }^{1}$, Santhana Eswara ${ }^{1}$ and Tom Wirtz ${ }^{1}$ \\ 1. Advanced Instrumentation for Ion Nano-Analytics (AINA), MRT Department, Luxembourg Institute \\ of Science and Technology (LIST), Belvaux, Luxembourg.
}

The investigation of nanostructured samples found in many fields of science like materials research and biology, poses significant challenges both in terms of spatial resolution and chemical sensitivity [1]. Often, a combination of different imaging and analysis techniques is required to establish a comprehensive picture of the sample and its properties. Correlative microscopy (CM) is being increasingly recognized as a powerful approach in both materials and life sciences to synergize the strengths and to overcome the inherent limitations of individual techniques. For example, the correlative light electron microscopy (CLEM) method combines live-cell imaging using low-resolution light microscopy to track fluorescent proteins and subsequent high resolution electron microscopy to establish structure function correlations. Several other correlative studies combining a multitude of techniques have been reported in many disciplines of the life sciences. Recently we developed a method based on the combination of Electron Microscopy (EM) with Secondary Ion Mass Spectrometry (SIMS), which is a chemically sensitive technique with excellent detection limits.

Here, we present the methodological aspects of correlation and image-fusion to combine the highresolution ultrastructural images obtained using Electron Microscopy (e.g. SEM, HIM, TEM) with the inherently lower-resolution chemical images obtained using SIMS. We demonstrate the method with different case-studies selected to broadly represent the typical samples in life science and materials science. More specifically, electron microscopy sharpening (EM-sharpening) which is closely related to "Pan-sharpening" is employed for fusing the images such that the color indicates the chemical information while the intensity is derived from high-resolution ultrastructural images [2]. We have also investigated alternative approaches to image fusion involving pixel-based and frequency-domain based methods. Practical issues such as those pertaining to the requirements of the raw data are highlighted. We will present and discuss both physical and technical aspects of correlative image overlay and imagefusion specific to SIMS-based correlative microscopy, including their applicability, advantages and disadvantages, in the context of 2D imaging [3-4]. 
References:

[1] T. Wirtz et al, Nanotechnology 26 (2015), p. 434001.

[2] J. G. Tarolli, L. M. Jackson and N. Winograd N., J. of The American Society for Mass Spectrometry 25 (2016), p. 2154.

[3] F. Vollnhals et al, Anal. Chem. 89 (2017), p. 10702.

[4] P. Gratia et al, ACS Energy Lett. 2 (2017), p. 2686.

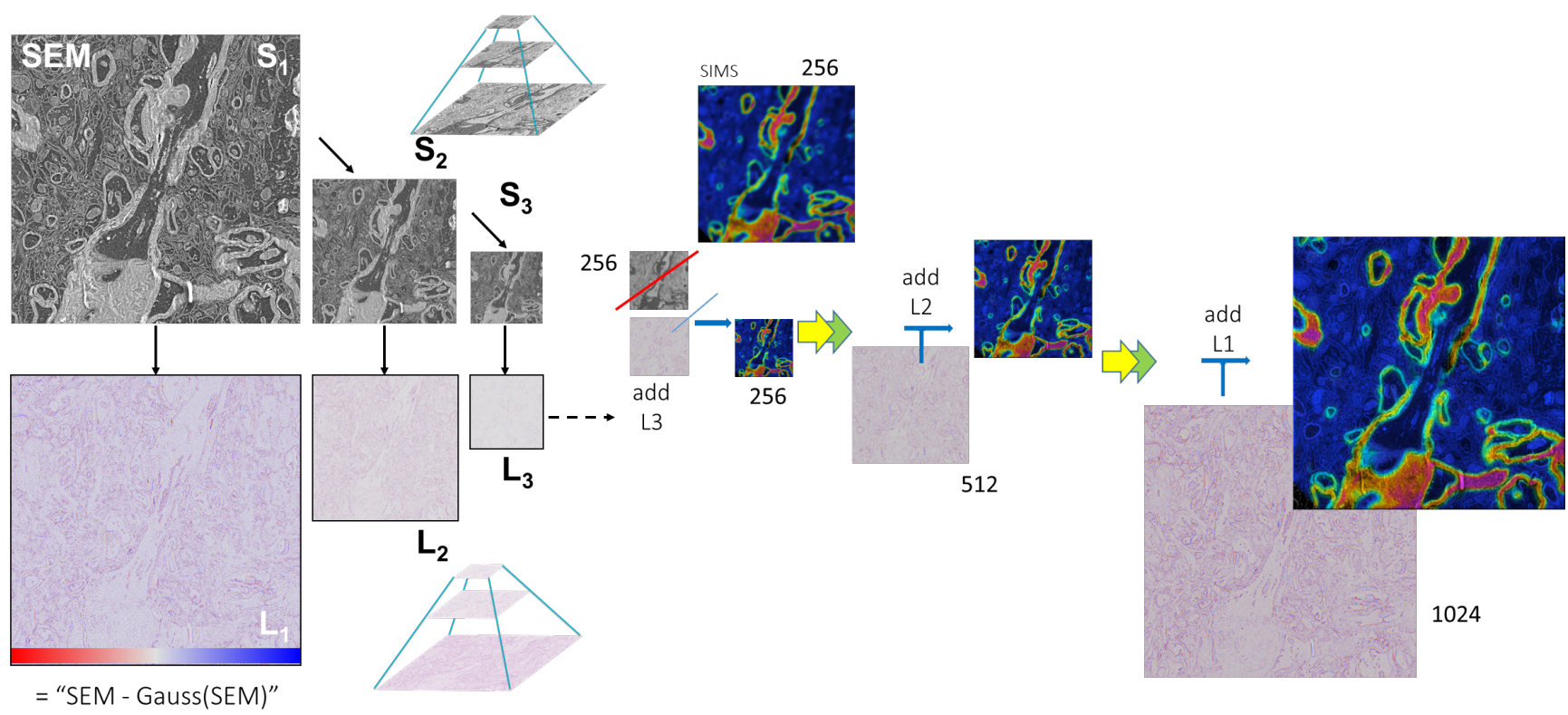

Figure 1. Illustration of the Laplace Fusion principle. Left: Construction of Laplacian pyramid (L1-L3) from input high-resolution image $(\mathrm{SEM}=\mathrm{S} 1)$ via Gaussfiltering, subtraction, and subsampling. The input SIMS image (center) is the brightness channel of an HSB transformed RGB image (IHS-LPF hybrid fusion). Right: Repeated steps of summation of Laplacian and input image followed by upsampling generate the sharpened version of the image. HSB to RGB transformation yields the final result Laplace fusion (EM+SIMS). 